\title{
First demonstration of real-time kinetic equilibrium reconstruction on TCV by coupling LIUQE and RAPTOR
}

\author{
F.Carpanese, F.Felici, C.Galperti, A.Merle, J.M.Moret, \\ O.Sauter and the TCV team $\ddagger$ \\ École Polytechnique Fédérale de Lausanne (EPFL), Swiss Plasma Center (SPC), CH- \\ 1015 Lausanne, Switzerland
}

\begin{abstract}
Kinetic Equilibrium Reconstruction (KER) in tokamaks is the solution of the free-boundary MHD equilibrium that best fits the external magnetic measurements and the internal plasma profiles as imposed from modeling and/or available internal measurements. In this paper, for the first time, the KER has been performed in real-time by coupling the free-boundary equilibrium code LIUQE to the 1.5D transport code RAPTOR, during a TCV discharge. The transport code is used as a dynamic state observer evolving the current diffusion equation, the electron heat and particle diffusion equations and correcting the prediction with the measurements available in real-time, making use of the Extended Kalman Filter (EKF) method. The simple coupling strategy, based on a matching of the free functions $\left(p^{\prime}\right.$ and $\left.T T^{\prime}\right)$ on the right-hand-side of the Grad Shafranov equation, is shown to be effective in providing consistency of pressure and current density profiles between the two codes. We also show that, despite this highly non-linear system, this technique does not require significant additional computational time with respect to the running times of the two independent codes and does not require a tight coupling of the two codes allowing therefore their independent development. The KER is compared to standard real-time equilibrium reconstruction, performed routinely in TCV, where only the external magnetic measurements are taken into account. The following cases are considered: flat central $n_{e}$, current density broadening due to applied electron cyclotron current drive and neoclassical tearing mode causing a temperature drop. The KER reproduces the expected changes of the internal profiles, tracking their dynamic evolution in their physical timescales.
\end{abstract}

Keywords: kinetic equilibrium reconstruction, real-time, free-boundary equilibrium, LIUQE, RAPTOR, tokamak, inverse problem

Submitted to: Nucl. Fusion

\section{Introduction}

A plasma state reconstruction problem aims to identify the plasma state from the available measurements meaning, in this work, the 2D flux map, the internal plasma current density and kinetic radial profiles. Magnetic Equilibrium Reconstruction

$\ddagger$ See author list of S. Coda et al 2019 Nucl. Fusion 59112023 
(MER) in tokamaks is the solution of the axisymmetric force balance, the GradShafranov equation (GSE) [1,2], that minimizes the least-squares error with the external magnetic measurements. This fundamental analysis is routinely performed in all tokamaks both during and after the experiment, being the starting point of almost every in-depth investigation (transport analysis, MHD stability analysis, fitting of diagnostic measurements to obtain 1D profiles, real-time control of plasma shape and position...). Many codes have been developed in different laboratories for this task [3-8]. In practice the internal pressure $p(\rho)$ and current density $j(\rho)$ profiles, where $\rho$ is a radial flux surface coordinate, entering in the Grad-Shafranov equation as two free functions, are expanded in terms of basis functions. The coefficients are found by solving a minimization problem for the least-squares error between the solution and the magnetic measurements. This problem is known to be numerically illconditioned: the external measurements are not very sensitive to coefficients affecting mainly internal profile features. In MER, this numerical issue is addressed either by reducing the number of basis functions or by imposing spatial smoothness. As a result, the reconstructed radial profiles are dependent on the regularization assumptions or on the choice of the basis functions degrading the fidelity of the reconstructed plasma state [9]. Kinetic Equilibrium Reconstruction (KER) overcomes this problem by providing direct constraints on the internal pressure and current density profiles from modeling and/or internal measurements when solving the GSE.

While MER in the past decades has converged to a standard and widely recognized approach, the KER is still an open field of research where many solutions with increasing level of complexity have been used and are still evolving. Indeed the name itself of "Kinetic Equilibrium Reconstruction" is not widely recognized, sometimes it can be referred as "integrated modeling" [10] or "integrated data analysis" [11,12] . For simplicity we will use the label KER in the rest of this paper.

A basic approach to perform a KER is constraining $p$ and $j$-related profiles from direct measurements of internal plasma quantities $\left(T_{e}, n_{e}, T_{i}, Z_{\text {eff }}\right.$, magnetic field pitch angle) [7, 13-15]. However the diagnostics for internal measurements, compared to the external magnetic measurements, have typically larger error-bars, might not cover the full radial profile and/or have operational limits [16] that prevent their routine use in all the experiments and during the full plasma discharge. This is the case in particular for the Motional Stark Effect (MSE) and polarimetry $[17,18]$ measurements, the only diagnostics providing information about the current distribution in the interior of the plasma in present-day tokamaks. Therefore, this approach has practical constraints in real-time applications and it is not routinely performed in most tokamaks.

A more advanced approach integrates dynamical models to supplement unavailable/unreliable/noisy measurements. This complements information from measurements with information about the (known) physics that governs the plasma state evolution, and helps reducing the effect of systematic errors and measurement noise on the reconstructed plasma state [19] . Focusing on the $j$ and $p$ evolution, $1 \mathrm{D}$ radial current diffusion equation $(\mathrm{CDE})$, heat diffusion, particle and impurity transport can be integrated. The increasing complexity naturally requires to face new challenges. For transport modeling, this means providing the model parameters (diffusion coefficient, conductivity...) and transport sources; find a strategy to combine multiple and eventually redundant diagnostics together with the model estimation; propagating the uncertainties due to both measurement inaccuracies and/or incomplete physical modeling. Also, the transport equations, the source 
models, as well as the spatial localization of diagnostic data, require geometrical information from the Grad-Shafranov equilibrium solution, hence a self-consistent coupling of all components of KER is required. It is important to remark that the basic approach is the reconstruction of time independent states (time slices) where the profile information are fed to the force balance equation from the available diagnostics for each time step individually. Introducing dynamical (time-dependent) models instead makes the approach a self-consistent dynamic state reconstruction of the flux map $\psi(R, Z, t), p(\rho, t)$ and $j(\rho, t)$ time evolution.

Several tools with different levels of complexity have been developed with this purpose. The "Integrated Data analysis Equilibrium" (IDE) [20], developed at ASDEX-Upgrade, couples the free boundary equilibrium with the current diffusion equation. A Bayesian framework called Integrated Data Analysis (IDA) [11] is used to combine internal kinetic measurements from several diagnostics, while propagating measurements uncertainties. The integrated modeling with "One Modeling Framework for Integrated Tasks" (OMFIT) [10] performs KER with transport codes (TRANSP [21], ONETWO [22]) including self-consistent equilibrium (EFIT [15]) and CDE solution and it is routinely used in several tokamaks. In TCV [23] the free boundary equilibrium reconstruction code LIUQE [3] has been coupled with the transport code ASTRA [24] and used to test the coupling technique [25] presented in this paper .

All the tools listed above have been developed to perform analysis after the experiments, however, in order to reliably achieve and maintain the performances required for future tokamaks such as ITER, real-time control of the kinetic and current density profiles, as well as the plasma equilibrium, will be needed [26, 27]. Not only control algorithms, but also supervisory control functions such as real-time plasma monitoring for disruption avoidance rely on the quality of the reconstruction of the plasma state [28]. Therefore, improving both the real-time reconstruction of internal profiles and plasma equilibrium with consistent profiles will be useful for plasma operation especially during high performance scenarios. In this paper we do not discuss possible improvements to real-time controllers nor supervisory control. We aim to demonstrate the achievement of a real-time KER: closing the loop between equilibrium and transport evolution in real-time, which is demonstrated here for the first time.

Implementing a real-time KER requires to fulfill extra constraints with respect to the off-line implementation:

- robustness against diagnostic failure/degradation

- fewer diagnostics are available in real-time or require computationally expensive post processing or human intervention

- computational time must be smaller than the characteristic time of the phenomena of interest in order to allow the possibility of reacting to plasma state changes and control the plasma.

A challenge to enable real-time kinetic equilibrium reconstruction, is to be able to solve the equations that govern key aspects of the physics of the tokamak evolution in real-time $[5,19,26]$. This presents a departure from traditional aims of physicsoriented codes in terms of accuracy and completeness. Control-oriented codes that are 'faster-than-real-time' have been developed for this purpose. Grad-Shafranov solvers aimed at real-time implementation are optimized to satisfy the computational time requirements [3] e.g. via parallelization and use of GPUs $[29,30]$. For the transport 
equations instead, efforts are made to reduce the complexity of the modeling while retaining the most relevant features [19,31,32], as well as using machine learning techniques to emulate the solutions of the most computational expensive part of the model [33-37]. Another approach is to entirely substitute physics-based models with data driven models trained on databases of previous experiments [38].

Taking into account the previous considerations for the existing frameworks and the real-time constraints, we adopt the following strategy in this paper:

- reduced first-principle-based dynamic models of plasma magnetic and thermal transport to supplement failing/missing diagnostics,

- combining modeling and measurements for the equations with an Extended Kalman Filter (EKF),

- Grad-Shafranov equilibrium with constrained internal profiles from the transport equations.

Other approaches are being investigated for this purpose, see for example [39], but their application is beyond the scope of this work.

To implement the chosen strategy, we performed a two-way coupling between the free boundary equilibrium reconstruction code LIUQE [3] and the transport code RAPTOR [40], both capable of real-time computational performances, through a matching of the free functions ( $p^{\prime}$ and $\left.T T^{\prime}\right)$ on the right-hand side of the GS equation. The RAPTOR [40] code is used to evolve the current diffusion equation, and provides LIUQE its only information about the internal current density profile (since no internal $j$ measurements are present on TCV). The electron heat diffusion equation is also solved by RAPTOR and combined with real-time measurements to provide $T_{e}$. The ion heat diffusion equation is here replaced by the prescription that $T_{i}$ is a fraction of $T_{e}$, but it can also be solved optionally. The particle diffusion for the electron density $n_{e}$ is solved by the RAPDENS [41] code (related to RAPTOR) and combined with real-time measurements from interferometers.

In summary, a real-time KER providing better estimation of the plasma state is an important step to improve real-time control of plasma performance and plasma supervision. In this paper we aim to:

- develop a coupling technique that guarantees consistency between the profiles of the magnetic equilibrium representation and of the real-time transport solver constrained by measurements,

- show the capability of running the coupled scheme in real-time during TCV discharges,

- prove that the real-time KER correctly reconstructs the expected time evolution of internal plasma features during different plasma scenarios and/or events.

With these aims in mind, the remainder of this paper is structured as follows: the KER implementation is presented in section 2, where the LIUQE equilibrium reconstruction code and the RAPTOR code used as dynamic state observer are briefly presented together with details on the real-time coupling strategy. In section 3 the first demonstration of the real-time KER obtained during TCV experiments is presented and compared to standard routinely computed real-time MER, showing in particular the capability of the former to estimate the physically expected internal profile modifications. In section 4 we summarize the limitations of the current approach and propose some ongoing improvements. We conclude in section 5. 


\section{Implementation of the Kinetic Equilibrium Reconstruction}

In this section the details of the implemented KER are presented, discussing first the free-boundary equilibrium reconstruction code LIUQE, then the transport code RAPTOR and finally the coupling strategy adopted in the real-time implementation. The main aim is to obtain an equilibrium solution with $p(\rho)$ and $j(\rho)$ constrained by kinetic measurements and modeling.

\subsection{Equilibrium reconstruction: LIUQE}

The Grad-Shafranov equation (GSE), namely the 2D MHD toroidal force balance equation under toroidal symmetry assumption [2] reads§)

$$
\Delta^{*} \psi=-4 \pi^{2} \mu_{0} R\left(R p_{L I U}^{\prime}+\frac{1}{\mu_{0} R} T T_{L I U}^{\prime}\right)
$$

where $\psi(R, Z)$ is the poloidal flux in cylindrical coordinates $(R, Z), p_{L U U}^{\prime} \equiv \frac{d p(\psi)}{d \psi}$ is the derivative of the total plasma pressure $p, T T_{L I U}^{\prime} \equiv T(\psi) \frac{d T(\psi)}{d \psi}$ with $T=R B_{\varphi}$ and $B_{\varphi}$ the toroidal component of the magnetic field. $p_{L I U}^{\prime}, T T_{L I U}^{\prime}$ are functions of the variable $\psi$ and it is convenient to introduce the label " $L I U$ " to explain the coupling technique in sec. 2.3. The elliptic operator is defined as $\Delta^{*} \equiv R \frac{\partial}{\partial R}\left(\frac{1}{R} \frac{\partial}{\partial R}\right)+\frac{\partial^{2}}{\partial Z^{2}}$.

In order to solve the equilibrium reconstruction problem, the free functions $p_{L I U}^{\prime}$ and $T T_{L I U}^{\prime}$ are expanded in the LIUQE code with basis functions series $p_{L I U}^{\prime}=$ $\sum_{i} \alpha_{i} A^{i}(\psi), T T_{L I U}^{\prime}=\sum_{i} \beta_{i} B^{i}(\psi)$. The coefficients $\left\{\alpha_{i}\right\},\left\{\beta_{i}\right\}$ are then sought by looking for the solution of GSE that minimizes the least-squares distance with external magnetic measurements, weighted by their uncertainties. Details of the available magnetic measurements in TCV and their relation with the solution of the GSE are found in $[3,43]$ together with the boundary conditions used in LIUQE. Note that LIUQE is fast enough to be used in real-time without additional assumptions with respect to the version used for post-shot equilibrium reconstruction, as detailed in [3], except for a reduced number of iterations to reach convergence.

As discussed already in the introduction, the problem to identify $\alpha_{i}, \beta_{i}$ from only external magnetic measurements is ill-posed, due to the little knowledge that these measurements themselves retain of the internal profile features. When performing standard MER, the problem is solved either by imposing some additional regularization to promote smooth solutions of $p_{L I U}^{\prime}, T T_{L I U}^{\prime}$ or by reducing the number of basis functions $\left\{A_{i}(\psi)\right\},\left\{B_{i}(\psi)\right\}$. The second option is adopted by LIUQE when performing the standard MER in TCV and will be used as a comparison to the KER in the results section 3. In particular, the MER typically considers only a unique linear in $\psi$ basis function for $A(\psi)$ and two basis functions for $\left\{B_{i}(\psi)\right\}$, linear and quadratic in $\psi$ respectively.

In the KER implemented in this paper instead of making ad-hoc assumptions on the basis functions we will provide direct constraints to the $p$ and $j_{\|}$profiles, as will be explained in sec. 2.3, where $j_{\|}=\langle\mathbf{j} \cdot \mathbf{B}\rangle / B_{0}$ is the flux surface average of the parallel component of the plasma current density $\mathbf{j}$ and $B_{0}$ is the vacuum toroidal magnetic field at the vessel mid-radius $R_{0}$. The choice of imposing constraints on $j_{\|}$, rather than on e.g. TT', will be natural when we will discuss the parallel Ohm's law in sec. 2.2 .

$\S$ assuming coordinate convention number $\operatorname{COCOS}=17$ [42] 
The relation between $p$ and $p_{L I U}^{\prime}$ is given by the definition itself $p_{L I U}^{\prime} \equiv \frac{d p(\psi)}{d \psi}$ while $j_{\|}$has the following dependence [19]:

$$
j_{\|}=-\frac{2 \pi T}{\mu_{0} B_{0}}\left\{\mu_{0} p_{L I U}^{\prime}+T T_{L I U}^{\prime}\left[g_{3}+\frac{g_{2}}{4 \pi^{2} T^{2}\left(V_{\hat{\rho}}^{\prime}\right)^{2}}\left(\frac{d \psi}{d \hat{\rho}}\right)^{2}\right]\right\},
$$

given $g_{2}=(d V / d \psi)^{2}\left\langle(\nabla \psi)^{2} / R^{2}\right\rangle, V$ the plasma volume, $g_{3}=\left\langle 1 / R^{2}\right\rangle, V_{\hat{\rho}}^{\prime}=d V / d \hat{\rho}$ with $\hat{\rho}=\sqrt{\Phi / \Phi_{b}}$ where $\Phi$ is the toroidal flux enclosed by a poloidal flux surface and $\Phi_{b}$ its value at the plasma boundary.

From the relations above it is clear that identifying $T T_{L I U}^{\prime}(\psi)$ and $p_{L I U}^{\prime}(\psi)$ by solving the reconstruction problem and finding $\left\{\alpha_{i}\right\},\left\{\beta_{i}\right\}$ means ultimately identifying $p(\psi)$ and $j_{\|}(\psi)$. Therefore, the hypothesis made on the basis functions in order to solve the issue of ill-posedness strongly affects the reconstruction of the internal plasma profiles.

\subsection{Radial transport: RAPTOR}

The KER implemented makes use of the real-time capable transport code RAPTOR as a dynamic state observer, to provide $p$ and $j_{\|}$information to the GSE. In this work the RAPTOR transport code evolves the flux surface averaged Ohm's law for $j_{\|}$and the radial electron temperature $T_{e}$ diffusion equation. A related code, called RAPDENS [41] includes a simplified model for the time evolution of the electron density profile $n_{e}$. RAPTOR and RAPDENS both make use of the Extended Kalman Filter technique to combine the prediction of the dynamic model with the estimation of the central $T_{e}$ from XTe (for RAPTOR) and the $n_{e}$ profile measurements from far infrared interferometer FIR [44] (for RAPDENS) [41, 45, 46].

In the remainder of this section we present, in order: the dynamic equations with details of model parameters and sources, the assumptions adopted for $n_{i}(\hat{\rho})$ and $T_{i}(\hat{\rho})$, some details on the EKF technique used to combine model and measurements and finally the details on the interface between RAPTOR and GSE.

2.2.1. Equations solved in RAPTOR A general quantity $X$ evolved in the flux surface averaged transport equation has the dependency $X=X(\hat{\rho}, t)$. The flux surface average Ohm's law, $j_{\|}=\sigma_{\|}<\mathbf{E} \cdot \mathbf{B} / B_{0}>+j_{n i}$ can be re-cast in terms of a poloidal flux diffusion equation [19] for $\psi(\hat{\rho}, t)$,

$$
\sigma_{\|} \frac{\partial \psi}{\partial t}=\frac{T^{2}}{16 \pi^{2} \mu_{0} \Phi_{b}^{2} \hat{\rho}} \frac{\partial}{\partial \hat{\rho}}\left(\frac{g_{2} g_{3}}{\hat{\rho}} \frac{\partial \psi}{\partial \hat{\rho}}\right)-\frac{B_{0}}{2 \Phi_{b} \hat{\rho}} V_{\hat{\rho}}^{\prime} j_{n i}
$$

where $\sigma_{\|}$is the neoclassical conductivity $[47,48]$ and $j_{n i}$ is the parallel flux surface averaged component of the non-inductive current density. We assume in this work that the toroidal field is fixed, the plasma shape is slowly changing and diamagnetic effects are small, therefore we expect a small contribution of the time varying term $\sigma_{\|} \frac{\hat{\rho} \dot{\Phi}_{b}}{2 \Phi_{b}} \frac{\partial \psi}{\partial \hat{\rho}}$ which is not included in equation (3) for simplicity. This term is implemented in RAPTOR, see [31] for full details, but not included during the simulations related to this paper.

In this paper $j_{n i}$ is composed of the bootstrap contribution $j_{b s}$ from Sauter's formula $[47,48]$ and the current driven by Electron Cyclotron Current Drive (ECCD) $j_{c d}$ given by a parametrized gaussian-like model [19],

$$
j_{c d}(\hat{\rho})=c_{c d} e^{\hat{\rho}^{2} / 0.5^{2}} \frac{T_{e}}{n_{e}} e^{-4\left(\hat{\rho}-\hat{\rho}_{d e p}\right)^{2} / w_{d e p}^{2}} P_{g}(t),
$$


where the central deposition location $\rho_{d e p}$ and width $w_{d e p}$ are prescribed, $P_{g}(t)$ is the power of each source of ECCD obtained in real-time, $n_{e}$ is computed from its transport equation and $c_{c d}$ is a machine-dependent proportionality factor. Other sources of current drive, such as neutral beam current drive, are not considered here since the experiments presented in sec. 3 only have ECCD.

The electron thermal energy diffusion equation reads,

$$
\frac{3}{2\left(V_{\hat{\rho}}^{\prime}\right)^{5 / 3}} \frac{\partial}{\partial t}\left[\left(V_{\hat{\rho}}^{\prime}\right)^{5 / 3} n_{e} T_{e}\right]=\frac{1}{V_{\hat{\rho}}^{\prime}} \frac{\partial}{\partial \hat{\rho}}\left[\frac{g_{1}}{V_{\hat{\rho}}^{\prime}} n_{e} \chi_{e} \frac{\partial T_{e}}{\partial \hat{\rho}}\right]+P_{e},
$$

defining the geometrical coefficient as $g_{1}=\left\langle(\nabla V)^{2}\right\rangle$. A negligible contribution of the electron particle flux $\Gamma_{e}$ to the heat diffusion equation (5) is assumed [31] and $n_{e}(\hat{\rho}, t)$ is given by the electron density diffusion equation specified later. In this work, for simplicity, an ad-hoc diffusivity coefficient $\chi_{e}$, tuned for TCV discharges has been used [19]. The total power density for electrons is written as $P_{e}=P_{O H}+P_{e, a u x}-P_{e i}-P_{e, r a d}$, where $P_{O H}$ is the Ohmic heating, $P_{e i}$ the power exchanged between electrons and ions and $P_{e, \text { rad }}$ the radiated power. In this work $P_{e, \text { aux }}$ is composed only by ECRH modeled with a Gaussian deposition of prescribed width and location.

The electron density diffusion equation, solved in RAPDENS module [41] within RAPTOR suite, reads

$$
\frac{\partial}{\partial t}\left[V_{\hat{\rho}}^{\prime} n_{e}\right]=-\frac{\partial \Gamma_{e}}{\partial \hat{\rho}}+V_{\hat{\rho}}^{\prime} S_{e}
$$

where the electron particle flux $\Gamma_{e}$ is given by,

$$
\Gamma_{e}=-\frac{g_{1}}{V_{\hat{\rho}}^{\prime}} D_{e} \frac{\partial n_{e}}{\partial \hat{\rho}}+g_{0} V_{e} n_{e},
$$

with $g_{0}=\langle\nabla V\rangle$, and the diffusion coefficient $D_{e}$ and pitch velocity $V_{e}$ are estimated to represent empirical system behavior. The model includes empirical expressions for thermal ionization of other neutral species, thermal recombination of ions, the particle sink in the scrape-off layer due to wall impact of particles exiting the plasma through the scrape-off layer. Details on the diffusion coefficient and particle sources are found in [41]. Like RAPTOR, RAPDENS uses an EKF approach merging the solution of the above model and the FIR [44] measurements to provide $n_{e}(\rho, t)$ which is then used as input in RAPTOR to solve the combined set of equations $(3,5)$ for $\psi$ and $T_{e}$.

The boundary conditions for the set of equations at $\hat{\rho}=0$,

$$
\left.\frac{\partial \psi}{\partial \hat{\rho}}\right|_{\hat{\rho}=0}=\left.\frac{\partial T_{e}}{\partial \hat{\rho}}\right|_{\hat{\rho}=0}=\left.\frac{\partial n_{e}}{\partial \hat{\rho}}\right|_{\hat{\rho}=0}=0
$$

and at the plasma boundary $\hat{\rho}=\hat{\rho}_{b}$

$$
\begin{aligned}
& \left.\left(\frac{g_{2}}{4 \pi^{2} \mu_{0}} \frac{1}{V_{\hat{\rho}}^{\prime}} \frac{\partial \psi}{\partial \hat{\rho}}\right)\right|_{\hat{\rho}=\hat{\rho}_{b}}=I_{p l}(t), \\
& \left.T_{e}\right|_{\hat{\rho}=\hat{\rho}_{b}}=\left.n_{e}\right|_{\hat{\rho}=\hat{\rho}_{b}}=0
\end{aligned}
$$

The ion temperature $T_{i}$ in this work is considered to be proportional to $T_{e}$ with a given ratio that can change in time for example when the electron cyclotron heating is applied. The ion density $n_{i}$ is computed in order to match the quasi-neutrality hypothesis assuming for TCV carbon as the main impurity and specifying the value of $Z_{\text {eff }}$. 
2.2.2. Extended Kalman Filter The Kalman Filter (KF) [49] is a tool to estimate the internal state $x$, corresponding to $T_{e}$ and $n_{e}$ independently in this work, of a dynamical system in real-time combining the model with the available measurements. In this work in particular the central $T_{e}$ value is estimated from the XTe diagnostic which uses ratios of soft X-ray channels and different line integrals of $n_{e}(\rho)$ are obtained from the FIR interferometer.

The method is designed to provide a recursive approximation of the state $x$ which maximizes its likelihood with respect to the available measurements. It requires a dynamic model $F$, capable of predicting the state at time $k$,

$$
x_{k}=F\left(x_{k-1}, v_{k-1}\right),
$$

given the state $x_{k-1}$ and the process noise $v_{k-1}$ at the previous time instant. In this case the model is the $T_{e}$ and $n_{e}$ diffusion equations.

Moreover, it requires a forward model $H$ for the diagnostic providing the expected measurement

$$
y_{k}=H\left(x_{k}, n_{k}\right)
$$

given the state $x_{k}$ and the observation noise $n_{k}$. In case of the FIR, for example, $H$ is the line integrated density for a given density profile and plasma equilibrium [41].

The approximation of the maximum-likelihood estimate of the state $\hat{x}_{k}$ is then given by

$$
\hat{x}_{k}=x_{k}+K_{k}\left(\tilde{y}_{k}-y_{k}\right),
$$

where $\tilde{y}_{k}$ is the actual measurement from the diagnostic, and $y_{k}$ is the expected measurements from the forward model as explained above. Equation (9) in particular combines a prediction $x_{k}$ from the model with a correction from the measurements $\tilde{y}_{k}$. The filter gain $K_{k}$ is designed to provide the minimum variance estimation, hence the maximum likelihood estimation, of the state $\hat{x}_{k}$, under the assumption of a linear system $F$ and considering the state $\hat{x}_{k}$ a random variable with Gaussian white noise $v$ and $n$. We refer to [50] for a full formulation of the problem.

To apply the filter to a non-linear system of equations the Extended Kalman Filter (EKF) technique propagates analytically the state variable $x$, with its Gaussian distribution, through the linearization of the non-linear system $F$. This way a suboptimal version of the KF is obtained meaning that EKF is not guaranteed to converge to the maximum likelihood estimation of the state in all the cases due to the linear approximation. However, the EKF is considered the de facto standard approach for estimation of states of a non-linear dynamical system in the system and control community.

We would like to point out that this approach has the double advantage of, on the one hand, correcting the predicted state that might be wrong due for example to the inaccurate knowledge of the transport coefficients, and on the other hand supplementing failing diagnostics with the model estimation. More generally, it could be applied to any forward model in the analysis, including the GS equation, and e.g. source models. However an analytic linearization of such models is not straightforward, while a numerical one would be computational expensive for real-time purposes. The details on the implementation of the EKF technique for the RAPTOR code can be found in [40].

In conclusion, the transport code RAPTOR receives $I_{p}$ from the magnetic measurements, the geometric information $g_{0, \ldots, 3}, V_{\hat{\rho}}^{\prime}$ and $\Phi_{b}$ from the equilibrium given by LIUQE. It evolves the flux diffusion equation, the electron heat and the particle 
diffusion equations combining the modeling with available measurements. From the solution of RAPTOR, equations (3) to (7), we can compute the total plasma pressure $p=n_{e} T_{e}+n_{i} T_{i}$ and

$$
j_{\|}=\frac{1}{8 \pi^{2} B_{0}} \frac{1}{\mu_{0} \Phi_{B}} \frac{T^{2}}{V_{\hat{\rho}}^{\prime}} \frac{\partial}{\partial \hat{\rho}}\left[\frac{g_{2} g_{3}}{\hat{\rho}} \frac{\partial \psi}{\partial \hat{\rho}}\right]
$$

from which one obtains $p_{R A P}^{\prime}=d p / d \psi$ and $T T_{R A P}^{\prime}$ inverting equation 2 with RAPTOR profiles:

$$
T T_{R A P}^{\prime}=\left(\mu_{0} p_{R A P}^{\prime}-j_{\|, R A P} \frac{\mu_{0} B_{0}}{2 \pi T}\right)\left[g_{3}+\frac{g_{2}}{4 \pi^{2} T^{2}\left(V_{\hat{\rho}}^{\prime}\right)^{2}}\left(\frac{d \psi}{d \hat{\rho}}\right)^{2}\right]^{-1},
$$

\subsection{Coupling methodology}

The coupling between the equilibrium and transport codes is explained in the following section. Instead of using polynomial basis functions, with regularization assumptions to solve the ill-posedness problem, we use $p_{R A P}^{\prime}\left(\psi_{N}\right), T T_{R A P}^{\prime}\left(\psi_{N}\right)$ as unique basis functions such that,

$$
\begin{aligned}
p_{L I U}^{\prime} & =\alpha p_{R A P}^{\prime}, \\
T T_{L I U}^{\prime} & =\beta T T_{R A P}^{\prime} .
\end{aligned}
$$

The least squares minimization problem with the external magnetic measurements needs to find only two coefficients. A full consistency of the two codes would require strictly $\alpha, \beta=1$. With our technique we expect $\alpha, \beta \approx 1$ whenever RAPTOR profiles are consistent with the magnetic measurements, obtaining in this case a good consistency of the profiles in the two codes. This assumption will be investigated in details in section 3.2 and the implication of this choice discussed further in this section.

The scheme is summarized in figure (1), in particular the closed loop symbolizes the fixed-point type of iteration to achieve consistency between the two codes. This is needed to solve the non-linearities in particular in the relation between $T T^{\prime}, j_{\|}$and the geometrical coefficients. We will show in sec. 3 that this coupling is sufficient to reach the goal of having $j_{\|}$and $p$ profiles matching between LIUQE and RAPTOR, whenever the magnetic measurements are consistent with the kinetic ones and the current diffusion modeling. The coupling is summarized in figure (1).

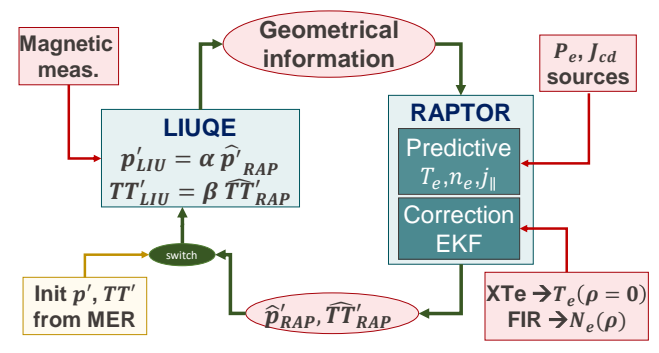

Figure 1. LIUQE/RAPTOR coupling schematics. Closed iteration loop indicated with green arrows. Inputs indicated with red arrows (color online). Initialization from MER in yellow.

In the real-time implementation, at every computational time step, one equilibrium solution (with $p_{R A P}^{\prime}, T T_{R A P}^{\prime}$ from the previous time step) and one 
transport solution (with the new equilibrium geometry) are performed. The convergence is reached after a few time steps. This is not a limitation since one cycle of the KER can be performed in less than 1.6ms (see sec. 3.1) whereas the current diffusion time in TCV is $100-200 \mathrm{~ms}$, the energy confinement time $\tau_{e}$ ranges from $2 \mathrm{~ms}$ to $50 \mathrm{~ms}$, the particle confinement time from $5 \tau_{e}$ to $10 \tau_{e}$. Convergence is therefore achieved also during time-evolving events of $j_{\|}$and $T_{e}$ simulated by their respective dynamic models in RAPTOR, as long as these changes follow the diffusive time scales. Moreover, bigger devices like ITER will have much longer time scales allowing eventually for multiple non-linear iterations for a single time step and/or a more refined spatial grid. A relaxation procedure, which in time acts as a low pass filter, is used to avoid the propagation of the noise in $p_{R A P}^{\prime}, T T_{R A P}^{\prime}$ (mostly originating from the measurements noise) into the LIUQE equilibrium solver, which helps convergence.

We would like to comment on the coupling strategy adopted. Using $p_{R A P}^{\prime}$ and $T T_{R A P}^{\prime}$ as basis functions has the following advantages: the codes LIUQE and RAPTOR required very little I/O modifications keeping them separated and allowing for their independent developments; with only two coefficients $\alpha$ and $\beta$ to be found, the least-squares problem is linear and did not increase the computational cost with respect to the standard MER, while remaining well-posed. In addition, the spatial details of the shape of the internal kinetic profiles depend only on the spatial resolution of the transport solver, without requiring a large number of basis function coefficients for the Grad-Shafranov equilibrium reconstruction. For example, reverse shear $q$ profiles can be represented in this way as well as steep pressure gradients and highly localized edge bootstrap current density in H-modes. One could, in principle, directly use $p_{L I U}^{\prime}=p_{R A P}^{\prime}$ and $T T_{L I U}^{\prime}=T T_{R A P}^{\prime}$, i.e. setting $\alpha, \beta=1$, avoiding to solve the minimization problem with the external magnetic measurements. In this case the magnetic measurements would not influence the reconstructed profiles. However uncertainty on the RAPTOR profiles is associated with a non-complete knowledge of correct transport models and to the simplification hypotheses adopted that will be extensively discussed in section 4 . Nevertheless, the magnetic measurements are known to retain information on the global scaling of these profiles. Hence, we preferred to leave $\alpha$ and $\beta$ free to be re-scaled in order to best fit the magnetic measurements. However, we will check a posteriori that $\alpha, \beta \approx 1$ which, as stated already at the beginning of this section, has to be intended as a confirmation that the profiles reconstructed by RAPTOR are consistent with the magnetic measurements.

Another solution could be to make use of a larger number of basis functions and introduce $j_{\|}$and $p$ as extra constraints on the least-squares minimization problem as in [20]. This approach has the advantage that the relative weights to be given to the extra constraints can be chosen with respect to the internal magnetic measurements depending on prior knowledge of their uncertainties, including eventually radially dependent weights. However, a disadvantage is that the size of the least-squares problem is increased if a large number of basis functions is required to represent spatial features of the profiles.

\section{Results of real-time KER in TCV}

The results of the real-time KER for the TCV discharge 62958 are presented in this section. The discharge, summarized in figure (2), was selected because several interesting physical events involving internal plasma profile evolution occurred (figure 
3). First, the initialization methodology and the computational cycle time achieved will be reported (sec. 3.1), then the effectiveness of the coupling methodology to provide the desired constraints to the equilibrium will be shown (sec. 3.2) and finally the results of the real-time KER will be compared to MER in presence of ECCD and during the formation and locking of an NTM (sec. 3.3).

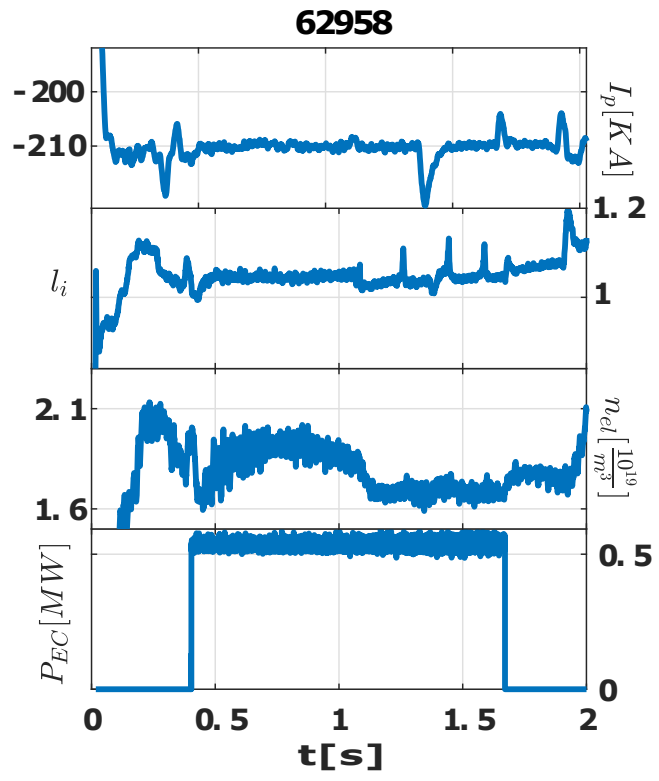

Figure 2. TCV discharge 62958 main physical parameters using standard off-line magnetic equilibrium reconstruction.

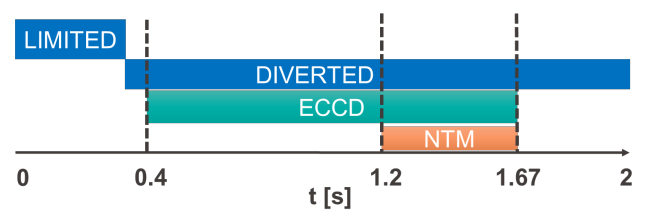

Figure 3. TCV discharge 62958 event intervals.

\subsection{Initialization and computational cycle time}

In previous work, the LIUQE and RAPTOR codes were implemented in Simulink and integrated in a node of the TCV plasma control system based on a i7-5930K $3.5 \mathrm{GHz}$ processor [51]. On this node, four threads are active, each running a different part of the algorithm. Thread 1 runs the density model with the EKF filter (RAPDENS) and two instances of LIUQE, the first performing the MER with standard basis functions and the second computing the KER with $p_{R A P}^{\prime}$ and $T T_{R A P}^{\prime}$ as basis functions. Both instances evaluate also the geometrical quantities (flux surface integrals) needed for the transport code. Thread 2 is dedicated to solve Ohm's law and the electron 
heat diffusion equation with RAPTOR. The remaining threads were not used for the simulation in this paper. LIUQE solves the GSE based on a finite difference approximation, it uses a spatial $(\mathrm{R}, \mathrm{Z})$ grid of $28 \times 65$ points to match the TCV elongated aspect ratio and 17 points on $\rho_{\psi}$ for the flux surface averaged integrals $g_{0, \ldots, 3}, T\left(\rho_{\psi}\right)$ and $V_{\hat{\rho}}^{\prime}$ computed using 32 values of the poloidal angle. The linear minimization problem includes all 133 available measurements and $\sim 60$ free parameters including the vessel and active coil currents together with the two basis function coefficients $\alpha$, $\beta$. In RAPTOR, which solves the equations based on a finite element approximation, the $n_{e}$ equation is solved by the RAPDENS module using cubic splines with 4 knots packed at the edge [41], while $T_{e}$ and $\psi(\rho)$ are represented by cubic splines with 11 knots [19].

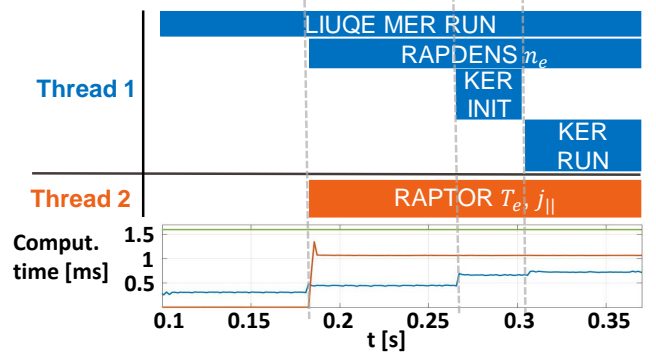

Figure 4. Shot 62958. Start-up of the coupling scheme up to closed loop convergence. Computational time of thread 1 (blue) and thread 2 (orange) during different tasks and sampling period communication between threads $1.6 \mathrm{~ms}$ (green).

The initialization procedure for the scheme (sec. 2.3) is shown in figure (4).

- The first instance of LIUQE, performing the standard MER, is initialized in thread 1.

- After waiting some time steps, to let the computation of the geometrical coefficients converge, RAPTOR starts running in thread $2(\sim 0.18 s)$ using the geometrical coefficients from LIUQE MER; when RAPTOR has relaxed from the initial conditions, the second instance of LIUQE, performing KER using $p_{R A P}^{\prime}$ and $T T_{R A P}^{\prime}$ as basis functions, is initialized $(\sim 0.27 s)$ from the last available solution of the MER.

- As for the MER also the KER instance of LIUQE requires some time steps for the geometrical coefficients to converge. Note that here we chose to let LIUQE evolve for approximately 18 iterations over $\sim 30 \mathrm{~ms}$. A smaller number of iterations would probably have sufficed however in this work we wanted to be sure to have a well converged geometrical coefficients before switching on the full coupling without investigating how early in the discharge we could have achieved it.

- At $\sim 0.31 s$ the loop is closed and the convergence is obtained after few iterations, i.e. RAPTOR uses geometrical information from the LIUQE KER, while providing $p_{R A P}^{\prime}$ and $T T_{R A P}^{\prime}$ for the next time step of LIUQE KER.

- Meanwhile, the standard LIUQE MER continues to run in background for comparison and as a restarting point in case of a failure of the coupling scheme.

The computational times are displayed in figure (4) (bottom). The communication period between threads was set to $\sim 1.6 \mathrm{~ms}$, thread 1 runs during the 
full coupling phase at $\sim 0.75 \mathrm{~ms}$ including two instances of LIUQE $(\sim 0.25 \mathrm{~ms}$ each) and the density observer $(\sim 0.15 \mathrm{~ms})$ while thread 2 runs in $\sim 1.1 \mathrm{~ms}$. Summing up all the computational times, considering only 1 LIUQE instance, the consistent KER could in principle run in a single thread in less than $1.6 \mathrm{~ms}$. Similar performances have been obtained in other discharges not reported in this work. A conservative and robust initialization procedure was deliberately adopted by waiting many time steps before switching to the full coupling since in this work we were interested in studying the performance of KER during the steady state phase without investigating the early plasma start-up phase. Nevertheless, the converged coupling is achieved before the transition from limited to diverted configuration at $\sim 0.31$ s and before the ECCD and NTM phases (see figure 3), and continues to work without failures till the end of the ECCD phase $(\sim 1.67 \mathrm{~s})$ and the plasma discharge.

During the analysis of the results, some erroneous settings of the real-time KER set-up were discovered due to earlier application of the code for other purposes, in particular the temperature ratio $T_{e} / T_{i}$ was set to 4 . This value is not the typically observed in TCV when the charge exchange radiation spectroscopy diagnostic is used to measure $T_{i}$ unless strong ECRH power is applied which is not the case for the discharges analyzed in this paper. Moreover the ECCD source in the current diffusion equation was turned off. Since these discharges were obtained at the end of the then running TCV experimental campaign, these shots could not be rerun. The quantitative results shown in the next section are therefore based on a re-simulation with the same real-time measurements, grid resolution and computer hardware but corrected transport model settings. In particular the $T_{e} / T_{i}$ was set to 1.42 during the ECCD phase and 1.2 otherwise, values that are within the typical bounds observed for this type of discharges. This modification does not affect the performance of the code, reproduces exactly how the code would have performed during the discharge and therefore does not invalidate the claims of this paper. We could have chosen to present the results of other experiments, however the erroneous settings were there during the full campaign and the physical content of the selected one was the most relevant to compare with MER.

\subsection{Investigating the coupling methodology results}

As a first result, we will prove that the coupling methodology detailed in section 2.3 is effective in providing $p(\rho, t)$ and $j_{\|}(\rho, t)$ constraints from measurements and modeling to the equilibrium solver. In figure (5) we compare the profiles reconstructed by RAPTOR KER (blue), with the ones compute by LIUQE KER (red) when $p_{R A P}^{\prime}$ and $T T_{R A P}^{\prime}$ are used as basis functions during three different experimental phases: at the beginning of the transition from limited to diverted shape (0.3s), during the ECCD phase $(0.8 \mathrm{~s})$ and finally when the NTM is fully developed $(1.4 \mathrm{~s})$. We find a good match for the quantities of interest, namely $p(\rho)$ and $j_{\|}(\rho)$ demonstrating consistent profiles in the equilibrium and transport codes. In particular, when the profiles from RAPTOR are consistent with the magnetic measurements, we expect that $\alpha, \beta \approx 1$ as is indeed the case in figure (6). Figure (5) aims only to show that the shape and amplitude of the profiles are coherent while a quantitative measure of them this is given directly by the coefficients in figure (6). We notice from figure (6) that a bigger discrepancy is found during the limited and NTM phases, indicating that the transport modeling is less accurate in these phases. We will elaborate further on this topic in section 4 . 

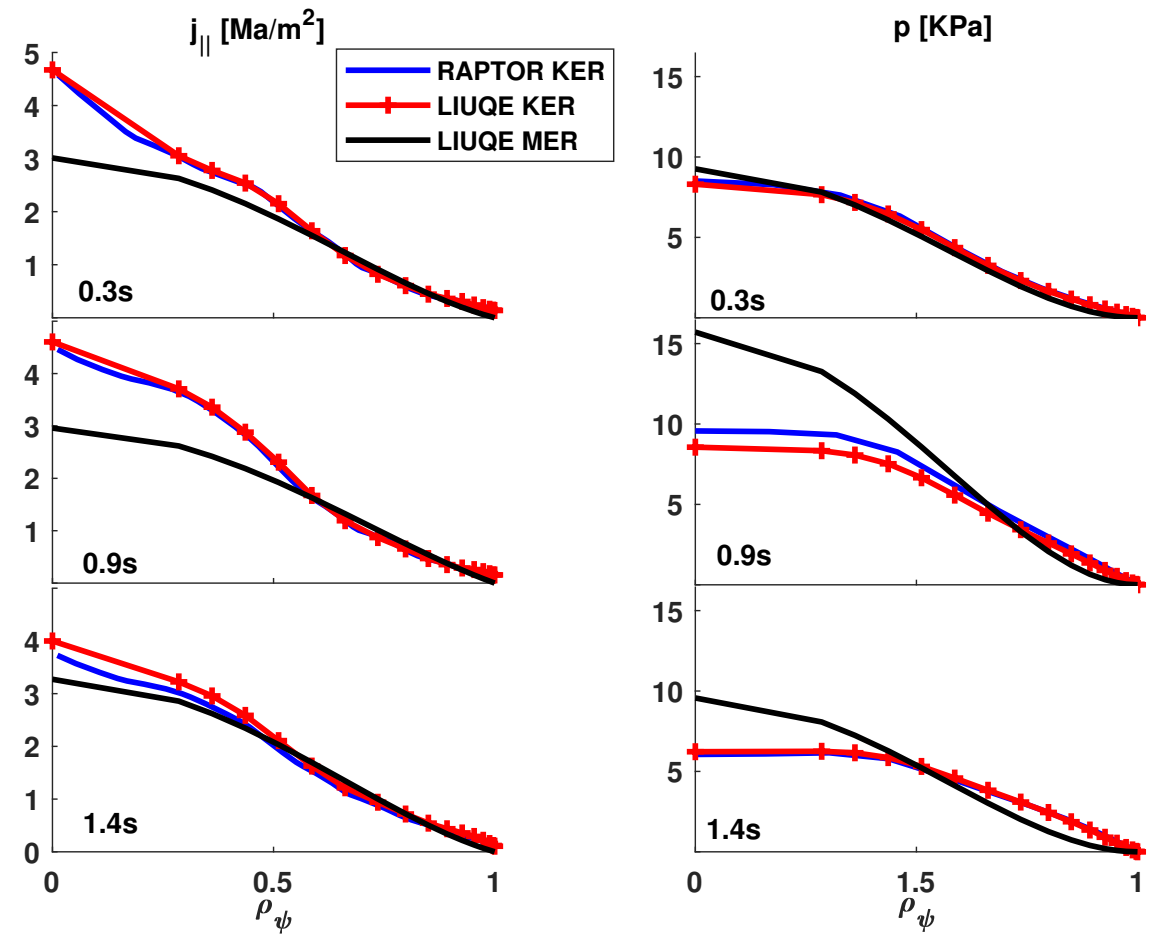

Figure 5. Shot 62958. Comparison of $j_{\|}$profile (left) and p profile (right) between RAPTOR KER(blue), LIUQE KER(red) and LIUQE MER(black) during three plasma phases: Ohmic diverted $(0.3 \mathrm{~s})$, off-axis current drive $(0.9 \mathrm{~s})$ and off-axis current drive in presence of NTM (1.4s).

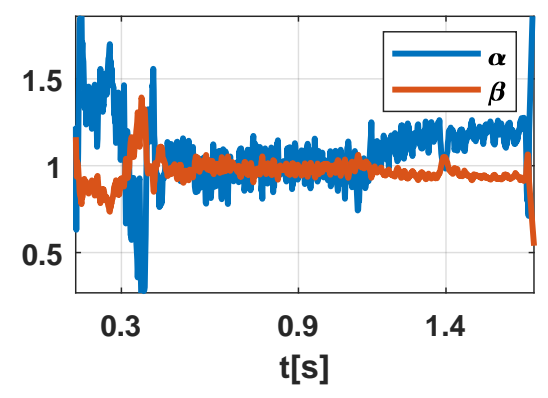

Figure 6. Shot 62958. $\alpha, \beta$ basis function coefficients

\subsection{Comparison between real-time MER and KER}

In this section we will show how the implemented KER, achieving real-time performances, is able to reconstruct well the expected internal profile features and profile dynamics due to particular physical events during the plasma discharges. Every time we will refer to MER in this section we will intend the standard real-time MER 
routinely computed during TCV operation. In the standard MER the set of basis functions has been chosen ( [3] and reference therein) in order to find a compromise between having a low error of the reconstruction with respect to the magnetic measurements and keeping the least squares minimization problem well-conditioned while avoiding under-fitting for a large range of experimental conditions. The KER, having constraints on the internal profiles coming from measurements/modeling, needs less a priori assumptions on the profiles. As expected, this allows the KER to better represent the true physical profiles. It is instructive to compare in this section the KER with the standard MER which was the only equilibrium reconstruction available in real-time so far for TCV.

We address first the contribution provided by the internal kinetic measurements to the reconstructed solution. In figure (5) the $p$ and $j_{\|}$profiles from KER (red cross) are compared to the MER (black). The central flattening of the pressure profile in KER at $0.9 \mathrm{~s}$ is due to the broad central density profile as shown in figure (7) and computed by RAPDENS (red) constrained in real-time by the FIR measurements. The flattening is confirmed by the post-shot Thomson scattering data (black) [52]. This flat feature is propagated to the equilibrium through $p_{R A P}^{\prime}$. The MER pressure profile instead has the dependence $p \propto\left(\rho^{2}-1\right)^{2}$, imposed by the basis functions adopted, hence it cannot reproduce the flattening. Given the consistency between the RAPTOR-KER

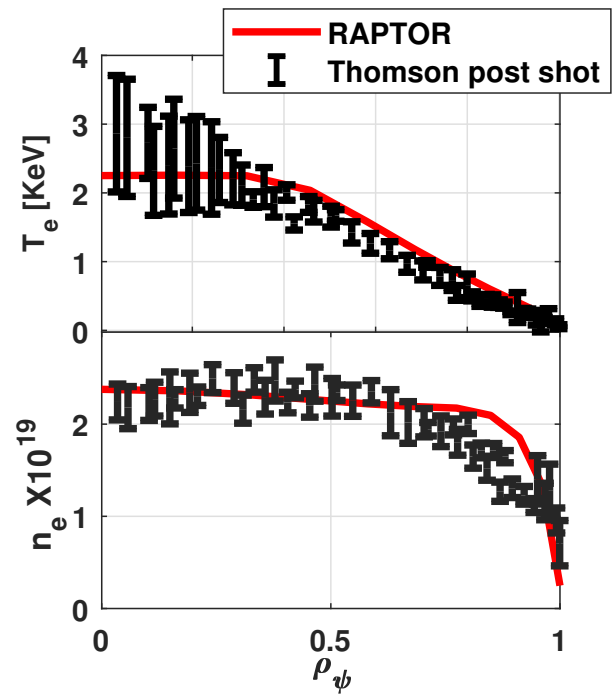

Figure 7. Shot 62958@0.9s. Comparison between $T_{e}$ and $n_{e}$ profiles reconstructed in real-time by RAPTOR/RAPDENS and the Thomson scattering measurements available after the experiment.

and LIUQE-KER profiles provided by the coupling and demonstrated in the previous section we will no longer make the distinction between them as in figure (5) (blue and red line) and we will consider from now on the LIUQE- KER to be representative of the reconstruction calling it simply with the label "KER".

We consider now the contribution provided by the $j_{\|}$constraint. A small off-axis external current drive contribution from ECCD with constant power and deposition location is added at $0.4 \mathrm{~s}$ while the total current is kept constant through feedback 
control of the central solenoid current. At $0.9 \mathrm{~s}$, when the current profiles are relaxed and in stationary condition, the different components of $j_{\|}$computed in real-time by RAPTOR are shown in figure (8). The small contribution from ECCD (green) produces a broadening of the total current density profile (red) while the contribution of the bootstrap current (violet) is very small. In figure (9) we compare the $j_{\|}$profile from KER and MER before the gyrotron is turned on (0.3s, dashed lines) and after when the current profile reaches a new stationary state $(0.9 \mathrm{~s}$, continuous lines). The profiles from KER (red and magenta) reproduce the expected broadening highlighted with the green zone between the arrows. The MER instead seems to be little affected by this contribution as a result of both the limited choice of basis functions and the low sensitivity of external magnetic measurements to small internal profile features. In this experiment the very small amount of externally driven current provided a small difference between MER and KER results localized only in the plasma center as found in figure (10) where the relative difference in percentage of the flux map $\Delta \psi(R, Z)=100 *\left(\psi_{M E R}-\psi_{K E R}\right) / \psi_{M E R}$ is displayed. A difference of $8 \%$ is found within the $\rho_{\psi_{N}}=0.5$ flux surface (blue line) and less than $1 \%$ everywhere else.

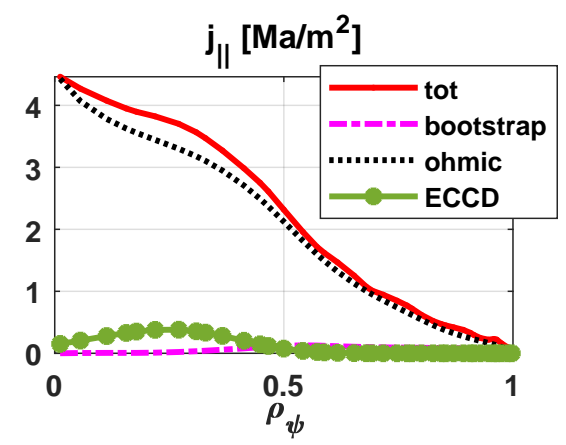

Figure 8. Shot 62958@0.9s. $j_{\|}$components computed by RAPTOR

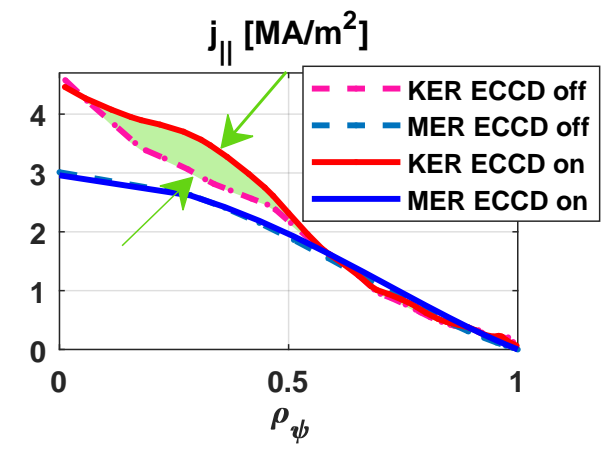

Figure 9. Shot 62958. Comparison of $j_{\|}$profiles between MER (blue, light blue) and KER (magenta,red) before (dashed lines, 0.3s) and during (continuous lines, $0.9 \mathrm{~s})$ the applied external ECCD.

The last result addresses the importance of including dynamical models together with the measurement constraints in order to reconstruct the time evolution of internal 


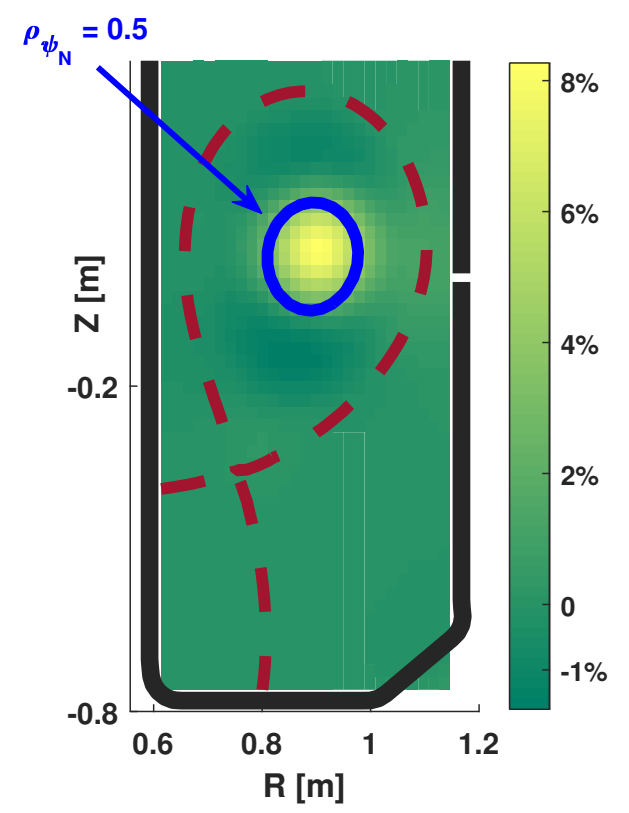

Figure 10. Shot 62958@0.9s. Relative poloidal flux map difference $\Delta \psi(R, Z)$ $=100 *\left(\psi_{K E R}-\psi_{M E R}\right) / \psi_{M E R}$ in percentage (green to yellow colormap), with $\rho_{\psi_{N}}=0.5$ surface (blue) and last close flux surface (red).

plasma profiles in their correct time-scales caused by physical events during the experiment. Thanks to the closed loop introduced (figure 1), the KER provides improvements to the reconstruction of single time instants, as shown above, but also to the full dynamic evolution as discussed in the remaining part of this section. From $t \sim 1.2 \mathrm{~s}$ an NTM develops causing the central temperature to slowly drop in $\sim 100 \mathrm{~ms}$ (figure 11). This evolution is detected in real-time by the XTe measurement of the central $T_{e}$ (green) and followed by the RAPTOR observer (red) in the correct time scale thanks to the EKF. As a direct consequence, the neoclassical conductivity $\sigma_{\|}$ in the Ohm's law, which scales as $\propto T_{e}^{3 / 2}$, drops in the central region $\rho_{\psi_{N}}<0.5$ (lines from red to yellow). The $j_{\|}$profile reconstructed by the KER follows this expected evolution. Indirectly this is also an example of a dynamical model, the current diffusion equation, that supplements the missing diagnostic for the internal plasma current density profile. The evolution of standard MER profiles (lines from blue to teal) are also shown for comparison.

\section{Limitations of current approach and outlooks}

We have shown that our KER approach can be implemented in real-time with very little modifications to both the equilibrium reconstruction and transport codes. The present technique has several limitations that are specific to practical constraints in TCV. We summarize them and propose improvements since they are typical of what is needed in present and future machines.

The electron density $n_{e}$ profile in the current implementation is computed by 


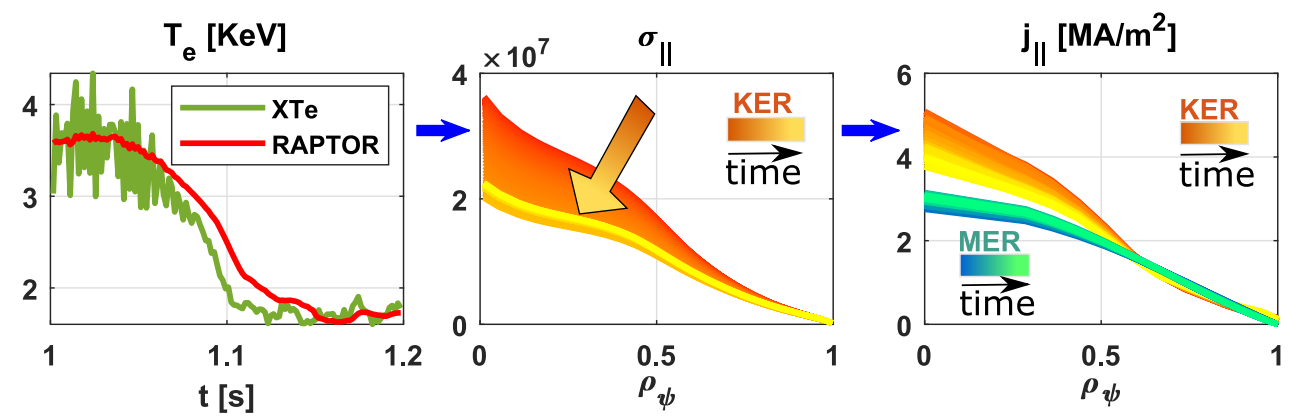

Figure 11. Shot 62958. From left to right: comparison of the evolution of central $T_{e}$ form real-time XTe and RAPTOR estimation during NTM formation and locking; evolution of neoclassical conductivity $\sigma_{\|}$during the event (from red to yellow); reconstructed evolution of $j_{\|}$from KER (red to yellow) and MER (blue to teal).

solving the diffusion equation constrained by the FIR measurement and fringe jumps can be avoided thanks to the model-based approach included in RAPDENS [41]. However using the FIR diagnostic is less precise than using Thomson scattering [52], in particular when non-monotonic or strong gradients are present. As an example in shot 62958 at $0.9 \mathrm{~s}$, the FIR measurements provides an edge steep gradient feature (figure 7 bottom) which is not confirmed by the Thomson scattering measurements.

The electron temperature $T_{e}$ profile in the current implementation is computed by solving the electron heat diffusion equation. Only the central value of the temperature is constrained by the real-time XTe estimation. Therefore, localized features (H-mode pedestal or flattening due to an internal mode) cannot be captured unless included somehow in the predictive modeling (which is the case for RAPTOR but this was not tested here).

Both $T_{e}$ and $n_{e}$ real-time reconstruction will benefit from the real-time Thomson scattering system, and such a system is planned to be commissioned in the coming campaign on TCV. Eventually an EKF technique will be implemented to combine the 1D diffusion modeling with the real-time Thomson measurements as done for the FIR in the current approach. In parallel, the predictive capability should be improved as well, in particular regarding the transport coefficients. RAPTOR can already include transport coefficients obtained from neural networks trained on gyrokinetic simulation results [34] and this should be included in our approach as well which will help applying it to other machines.

The parallel current density $\left(j_{\|}\right)$is obtained by solving the current diffusion equation (3). Unfortunately no internal measurements (MSE, polarimetry) are available at present in TCV neither to constrain the modeling nor to confirm it. In other tokamaks these diagnostics are used to constrain the equilibrium solution directly, also in real-time as in $[7,13-15,53,54]$. In principle, real-time MSE measurements can also be included in RAPTOR with the EKF technique as described in [27]. We favour this approach when eventually porting this tool to other tokamaks that have real-time MSE measurements.

An improvement on the description of the current sources can be obtained, for example making use of the real-time capable ray tracing code TORBEAM [55]. This code has already been coupled in real-time to RAPTOR in AUG and TCV and 
provides the real-time deposition location and deposited power of the ECRH sources. A real-time capable code for simulating the effects of NBI sources (RABBIT, [56]) will also be included in the future.

Another important aspect that has not been considered in the current implementation is the internal profile crash due to the presence of sawtooth instabilities. This leads, in the present implementation, to non-physical central safety factor much below 1. In [57] it was shown that the CDE coupled to the equilibrium solution is able to reproduce the measured q-profile evolution associated to a sawtooth crash, when sawtooth-induced current relaxation is accounted for by reconnection models [58], and the fast ions contribution is correctly modeled. These reconnection models are already implemented in RAPTOR [59] but were not used in this paper .

The fast particle contribution to the current drive and the total pressure has not been considered in the current approach, however the previously mentioned RABBIT code [56] includes these effects.

In the results presented in section 3 the $T_{i}$ profile is assumed to be proportional to $T_{e}$ and the proportionality constant is considered as a free-parameter that needs to be specified a priori. A sensitivity analysis revealed that the $j$ profile reconstructed by the transport solver is not very sensitive to this parameter, since mainly $T_{e}$ is important for $\sigma_{n e o}, j_{e c c d}$ and $j_{b s}$. However, a wrong choice of this parameter leads to a poor estimate of the total plasma pressure in the transport solver. To mitigate this problem, since the magnetic measurements retain information on the scaling of the total plasma pressure, we let $p^{\prime}$ free to be scaled in the equilibrium solver by the coefficient $\alpha$ in order to match the magnetic measurements in the least squares sense. Hence, $\alpha$ different than 1 indicates that the total plasma pressure of the transport solver is not consistent with the magnetic measurements. Another possible approach is to include the $T_{i}$ evolution equation in case a reliable transport modeling is available and/or benefit directly from CXRS real-time measurements. In the absence of that, another solution is to adjust in real-time the $T_{e} / T_{i}$ ratio in order to match the total stored kinetic energy $W_{k}$ between the equilibrium solution and transport solution. This approach is detailed in section 4.1 and was found very promising.

The $n_{i}$ profile is presently considered to be proportional to $n_{e}$ in order to respect the quasi-neutrality assumption, while providing a priori information on the effective charge $Z_{\text {eff }}$. No reliable direct measurements of this quantities are available in realtime so far. The loop voltage measurements can be used to estimate this quantity as in [60]. Models to study the radial impurity transport are under investigation but not yet sufficiently mature to consider their use in real-time.

To conclude this section we would like to comment on one of the most natural continuation of this work. In [27] the LIUQE and RAPTOR codes, without a selfconsistent coupling, were used in TCV to develop a model predictive control technique to control $\iota$ profile and $\beta$. In the present paper we did not close the loop with the real-time controllers yet. However, the difference between the $\iota$ profile between LIUQE and RAPTOR observed in [27] is now resolved thanks to the achieved KER. Moreover, the RAPTOR results depend on the geometrical coefficients given by LIUQE which non-linearly depend on the $\iota$ profile of the latter. Therefore, having consistent profiles in LIUQE removes one source of uncertainty for the $\iota$ estimation of RAPTOR which is the only estimate that the controllers rely on due the lack of direct measurements of the current density profile in TCV. For this low plasma current discharge the difference is not expected to be significant, but we argue that it might become important in case of hybrid or reversed shear scenarios for example. A dedicated investigation 
will be carried out on the influence of KER to the performance of the real-time profile controllers in particular for advanced scenarios. Note that the present RT-KER scheme will provide improved information for many RT-control tasks, for example the NTM control and performance control, as well as supervision of the time evolution of the discharge with respect to the predetermined expected trajectories.

\subsection{Estimate $T_{e} / T_{i}$ ratio from matching the total kinetic energy between equilibrium reconstruction and transport solution}

In the absence of real-time $T_{i}$ measurements and reliable transport modeling we propose in this section an approach to estimate the $T_{e} / T_{i}$ ratio within the implemented KER. This is based on the assumption that the magnetic measurements all together retain reliable information on global plasma parameters such as the total kinetic energy $W_{k}$, i.e. the volume integral of the total plasma pressure profile $p$. Moreover, among the free parameters to be selected in the transport simulation $\left(Z_{\text {eff }}\right.$, transport coefficients, ...), $T_{e} / T_{i}$ influences the most the estimate of the total pressure of the transport code, $T_{e}$ and $n_{e}$ being constrained by the available real-time measurements in our implementation.

As discussed in this paper, we consider $p_{L I U}^{\prime}=\alpha p_{R A P}^{\prime}, T T_{L I U}^{\prime}=\beta T T_{R A P}^{\prime}$. Again we let $\alpha, \beta$ be found in order for the equilibrium solution to best match the magnetic measurements. From the total plasma pressure of the equilibrium reconstruction $p_{L I U}$ we compute the total stored energy $W_{k, L I U}$. In this approach, differently to section 2.3, we add a PI (proportional integral) [61] controller that changes the $T_{e} / T_{i}$ input of the transport code such that $W_{k, R A P}$ matches $W_{k, L I U}$. In this way the magnetic measurements are used to constrain $W_{k, L I U}$ which is then used to estimate $T_{e} / T_{i}$ and ensure consistency of the total stored energy between the equilibrium and the transport codes. This approach removes the need to specify $T_{e} / T_{i}$ a priori.

In figure (12) we compare the results obtained with $T_{e} / T_{i}=4$, as used during the experiments, and an offline re-simulation using the technique described in this section. The top figure compares the total stored energy $W_{k}$ for the case of $T_{e} / T_{i}=4$ (black line LIUQE, blue line RAPTOR) and the estimation with the PI controller technique (green line LIUQE, red line RAPTOR). The middle figure shows the obtained $\alpha$ and $\beta$ coefficients, dark/light green for the case of $T_{e} / T_{i}=4$ and dark/light blue for the estimate with the technique explained in this section. The bottom figure shows the $T_{e} / T_{i}$ ratio obtained from the PI controller.

As a result the PI controller is effectively changing the $T_{e} / T_{i}$ ratio in order to make RAPTOR's $W_{k}$ (red line top figure) match the LIUQE one (green line figure). This results in $\alpha, \beta$ coefficients of the simulation using this technique (dark/light blue middle figure) closer to 1 with respect to the case $T_{e} / T_{i}=4$ (dark/light green middle figure). This confirms that the value $T_{e} / T_{i}=4$ used during the TCV discharge provided pressure profiles not consistent with magnetic measurements.

The controller is forced to provide $T_{e} / T_{i}$ between 1 and 4 , the typical values found in TCV at least for cases without direct ion heating as for the discharge presented in this paper. In particular we do not expect $T_{e}<T_{i}$ since in this discharge we expect dominant electron heating. During the first $0.3 \mathrm{~s}$ the controller would request a ratio lower than the minimum bound, explaining the poor matching of $W_{k}$ during this time interval.

In section 3 we chose to fix $T_{e} / T_{i}$ ratio equal to 1.2 during the ECCD phase and 1.4 otherwise (from comparison with offline simulations) which provided results 
similar to the ones obtained with the technique proposed in this section.

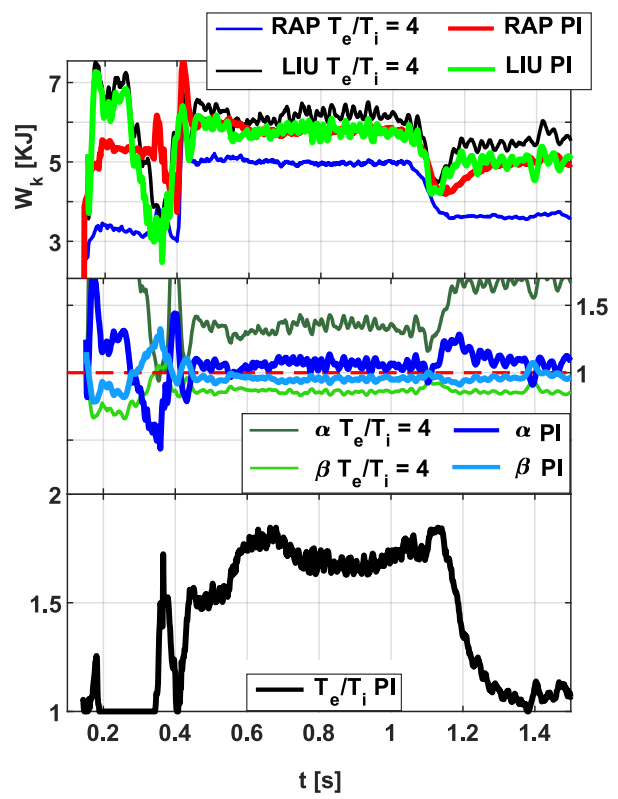

Figure 12. Shot 62958. Comparison between KER with constant $T_{e} / T_{i}=4$ and with $T_{e} / T_{i}$ controlled by PI controller aiming at $W_{k}$ consistency between LIUQE and RAPTOR as explained in section 4.1. Top figure: $W_{k}$ comparison between $T_{e} / T_{i}=4$ (blue RAPTOR, black LIUQE) and with PI controller (red RAPTOR, green LIUQE). Middle figure: $\alpha, \beta$ comparison between $T_{e} / T_{i}=4$ case (dark/light green) and re-simulation with PI controller (dark/light blue). Bottom figure: $T_{e} / T_{i}$ estimate from PI controller case.

As a last caveat we would like to point out that magnetic measurements are sensitive to the total plasma pressure including supra-thermal components and thus $W_{k, L I U}$ can contain a supra-thermal contribution as well. Without a proper description of those components in the transport model, this method could still be used yielding an effective ion temperature but at the cost of a reduced fidelity in particular when computing quantities depending on $T_{i}$ such as the energy transfer between ions and electrons or the boostrap current. Additionally these supra-thermal pressure components could be anisotropic whereas LIUQE assumes an isotropic pressure. No significant supra-thermal pressure nor anisotropy are present in the discharge presented here.

\section{Conclusions}

Improving the real-time plasma state reconstruction, in particular the flux distribution and internal plasma profiles, is an important first step to allow plasma supervision and reliable control of performances. With this aim, we presented in this paper the first kinetic equilibrium reconstruction (KER) performed in real-time with profiles constrained by a real-time transport model, achieving a computational time of less than $1.6 \mathrm{~ms}$, faster than the current diffusion time and electron confinement time of 
the TCV tokamak.

The approach consists in coupling the free boundary equilibrium reconstruction code LIUQE and the radial transport code RAPTOR. The transport code evolves predictively the $T_{e}, n_{e}$ and $j_{\|} 1 \mathrm{D}$ diffusion equations together with their external sources, making use of the Extended Kalman Filter technique to correct the modeling using the measurements available in real-time. The equilibrium code receives the information of $p(\rho, t)$ and $j_{\|}(\rho, t)$ from the transport code through the computed $p^{\prime}(\rho, t)$ and $T T^{\prime}(\rho, t)$ used as basis functions on the right-hand-side of the GradShafranov equation. LIUQE then provides geometrical information to the radial transport equations, closing the feedback loop between the two codes.

The simple coupling technique proposed in this paper does not require significant additional computational time with respect to the one required to run the two codes independently. Furthermore, our results show that a tight coupling between the two codes is not necessary, allowing their independent development. Moreover, it proves to correctly provide $p$ and $j_{\|}$constraints to the equilibrium solver, converging in a few iterations and allowing therefore real-time performances. To our knowledge, this is the first time that a KER is performed in real-time during tokamak operation including consistently the solution of a real-time transport model.

We compared the results of the KER with the real-time standard equilibrium reconstruction routinely performed in TCV, which considers external magnetic measurements only, for cases where we expected internal plasma profile modifications due to physical events. In all cases the implemented KER was able to reproduce these modifications, improving not only the reconstruction on a single time instant but also allowing the equilibrium solution to follow the time evolution of internal profiles driven by the transport modeling and real-time measurements.

The limitations of the approach together with the improvements expected in future developments have been discussed. So far the KER has been tested only in low power discharges in TCV where, as expected, no strong differences have been found compared to MER. The approach will be tested on more reactor-relevant discharges in the upcoming TCV campaigns and compared with more refined integrated modeling available during the post-shot analysis. After this investigation, the real-time KER will be coupled to the kinetic and magnetic real-time controllers.

\section{Acknowlegments}

This work has been carried out within the framework of the EUROfusion Consortium and has received funding from the Euratom research and training programme 20142018 and 20192020 under Grant agreement No. 633053. The views and opinions expressed herein do not necessarily reflect those of the European Commission. This work was supported in part by the Swiss National Science Foundation.

\section{References}

[1] H. Grad and H. Rubin, "Hydromagnetic equilibria and force-free fields," Journal of Nuclear Energy (1954), vol. 7, no. 3-4, pp. 284-285, 1958.

[2] V. Shafranov, "Plasma equilibrium in a magnetic field," Reviews of plasma physics, vol. 2, p. $103,1966$.

[3] J.-M. Moret, B. Duval, H. Le, S. Coda, F. Felici, and H. Reimerdes, "Tokamak equilibrium reconstruction code liuqe and its real time implementation," Fusion Engineering and Design, vol. 91, pp. 1-15, 2015. 
[4] P. J. McCarthy, P. Martin, and W. Schneider, "The CLISTE interpretive equilibrium code," Max-Planck-Institut für Plasmaphysik, 1999.

[5] J. Ferron, M. Walker, L. Lao, H. S. John, D. Humphreys, and J. Leuer, "Real time equilibrium reconstruction for tokamak discharge control," Nuclear Fusion, vol. 38, no. 7, p. 1055, 1998.

[6] J. Blum, C. Boulbe, and B. Faugeras, "Reconstruction of the equilibrium of the plasma in a tokamak and identification of the current density profile in real time," Journal of computational physics, vol. 231, no. 3, pp. 960-980, 2012.

[7] L. Lao, H. S. John, Q. Peng, J. Ferron, E. Strait, T. Taylor, W. Meyer, C. Zhang, and K. You, "Mhd equilibrium reconstruction in the DIII-D tokamak," Fusion science and technology, vol. 48, no. 2, pp. 968-977, 2005.

[8] L. Giannone, R. Fischer, P. McCarthy, T. Odstrcil, I. Zammuto, A. Bock, G. Conway, J. Fuchs, A. Gude, V. Igochine, et al., "Improvements for real-time magnetic equilibrium reconstruction on asdex upgrade," Fusion Engineering and Design, vol. 100, pp. 519-524, 2015.

[9] L. Zakharov, E. Foley, F. Levinton, and H. Yuh, "Reconstruction of the q and p profiles in iter from external and internal measurements," Plasma physics reports, vol. 34, no. 3, pp. 173-188, 2008.

[10] O. Meneghini, S. Smith, L. Lao, O. Izacard, Q. Ren, J. Park, J. Candy, Z. Wang, C. Luna, V. Izzo, et al., "Integrated modeling applications for tokamak experiments with omfit," Nuclear Fusion, vol. 55, no. 8, p. 083008, 2015.

[11] R. Fischer, C. Fuchs, B. Kurzan, W. Suttrop, E. Wolfrum, and A. U. Team, "Integrated data analysis of profile diagnostics at ASDEX upgrade," Fusion science and technology, vol. 58, no. 2, pp. 675-684, 2010.

[12] P. Garcia-Martinez and P. Montes, "An efficient mhd equilibrium solver for control oriented transport models," Fusion Engineering and Design, vol. 152, p. 111455, 2020.

[13] M. Brix, N. Hawkes, A. Boboc, V. Drozdov, S. Sharapov, and J.-E. Contributors, "Accuracy of EFIT equilibrium reconstruction with internal diagnostic information at jet," Review of Scientific Instruments, vol. 79, no. 10, p. 10F701, 2008.

[14] J. Qian, L. Lao, H. Liu, W. Ding, L. Zeng, Z. Luo, Q. Ren, Y. Huang, J. Huang, D. Brower, et al., "EAST equilibrium current profile reconstruction using polarimeter-interferometer internal measurement constraints," Nuclear Fusion, vol. 57, no. 3, p. 036008, 2016.

[15] Y. Li, P. Lotte, W. Zwingmann, C. Gil, and F. Imbeaux, "EFIT equilibrium reconstruction including polarimetry measurements on tore supra," Fusion Science and Technology, vol. 59, no. 2, pp. 397-405, 2011.

[16] A. Bock, E. Fable, R. Fischer, M. Reich, D. Rittich, J. Stober, M. Bernert, A. Burckhart, H. Doerk, M. Dunne, et al., "Non-inductive improved h-mode operation at asdex upgrade," Nuclear Fusion, vol. 57, no. 12, p. 126041, 2017.

[17] F. Levinton, R. Fonck, G. Gammel, R. Kaita, H. Kugel, E. Powell, and D. Roberts, "Magnetic field pitch-angle measurments in the pbx-m tokamak using the motional stark effect," Physical review letters, vol. 63, no. 19, p. 2060, 1989.

[18] D. Wroblewski and L. Lao, "Polarimetry of motional stark effect and determination of current profiles in diii-d," Review of scientific instruments, vol. 63, no. 10, pp. 5140-5147, 1992.

[19] F. Felici, O. Sauter, S. Coda, B. Duval, T. Goodman, J. Moret, J. Paley, T. Team, et al., "Real-time physics-model-based simulation of the current density profile in tokamak plasmas," Nuclear Fusion, vol. 51, no. 8, p. 083052, 2011.

[20] R. Fischer, A. Bock, M. Dunne, J. Fuchs, L. Giannone, K. Lackner, P. McCarthy, E. Poli, R. Preuss, M. Rampp, et al., "Coupling of the flux diffusion equation with the equilibrium reconstruction at ASDEX upgrade," Fusion Science and Technology, vol. 69, no. 2, pp. 526$536,2016$.

[21] R. Hawryluk, "An empirical approach to tokamak transport," pp. 19-46, Elsevier, 1981.

[22] L. Owen, J. Canik, R. Groebner, J. Callen, X. Bonnin, and T. Osborne, "Comparing 1.5d ONETWO and 2d SOLPS analyses of inter-ELM h-mode plasma in DIII-d," Nuclear Fusion, vol. 50, p. 064017, may 2010.

[23] S. Coda, J. Ahn, R. Albanese, S. Alberti, E. Alessi, S. Allan, H. Anand, G. Anastassiou, Y. Andrèbe, C. Angioni, et al., "Overview of the tcv tokamak program: scientific progress and facility upgrades," Nuclear Fusion, vol. 57, no. 10, p. 102011, 2017.

[24] G. V. Pereverzev and P. Yushmanov, "Astra. automated system for transport analysis in a tokamak," Max-Planck-Institut für Plasmaphysik report, 2002.

[25] F. Carpanese, O. Sauter, A. Merle, F. Felici, and J. Moret, "Kinetic equilibrium reconstruction on tcv: towards a self-consistent approach," 45th EPS Conference on Plasma Physics, 2018.

[26] D. Humphreys, G. Ambrosino, P. de Vries, F. Felici, S. H. Kim, G. Jackson, A. Kallenbach, E. Kolemen, J. Lister, D. Moreau, et al., "Novel aspects of plasma control in iter," Physics 
of Plasmas, vol. 22, no. 2, p. 021806, 2015.

[27] M. C. Messmer, F. Felici, O. Sauter, A. A. Teplukhina, J. P. Loenen, M. Reich, R. Fischer, D. Rittich, and R. J. Jaspers, "Optimal MSE polarisation angle and q-profile estimation using kalman filters and the plasma simulator RAPTOR," Plasma Physics and Controlled Fusion, 2018.

[28] T. C. Blanken, F. Felici, C. Galperti, N. M. T. Vu, M. Kong, O. Sauter, M. de Baar, E. M. Team, T. Team, et al., "Real-time plasma state monitoring and supervisory control on tcv," Nuclear Fusion, vol. 59, no. 2, p. 026017, 2019.

[29] Y. Huang, B.-J. Xiao, and Z.-P. Luo, "Fast parallel grad-shafranov solver for real-time equilibrium reconstruction in east tokamak using graphic processing unit," Chinese Physics B, vol. 26, no. 8, p. 085204, 2017.

[30] M. Rampp, R. Preuss, R. Fischer, and A. U. Team, "Gpec: A real-time-capable tokamak equilibrium code," Fusion Science and Technology, vol. 70, no. 1, pp. 1-13, 2016.

[31] A. Teplukhina, O. Sauter, F. Felici, A. Merle, D. Kim, T. Team, E. M. Team, et al., "Simulation of profile evolution from ramp-up to ramp-down and optimization of tokamak plasma termination with the raptor code," Plasma Physics and Controlled Fusion, vol. 59, no. 12, p. 124004, 2017.

[32] J. E. Barton, M. D. Boyer, W. Shi, W. Wehner, E. Schuster, J. R. Ferron, M. L. Walker, D. A. Humphreys, T. C. Luce, F. Turco, et al., "Physics-model-based nonlinear actuator trajectory optimization and safety factor profile feedback control for advanced scenario development in diii-d," Nuclear Fusion, vol. 55, no. 9, p. 093005, 2015.

[33] O. Meneghini, S. P. Smith, P. B. Snyder, G. M. Staebler, J. Candy, E. Belli, L. Lao, M. Kostuk, T. Luce, T. Luda, et al., "Self-consistent core-pedestal transport simulations with neural network accelerated models," Nuclear Fusion, vol. 57, no. 8, p. 086034, 2017.

[34] J. Citrin, S. Breton, F. Felici, F. Imbeaux, T. Aniel, J. Artaud, B. Baiocchi, C. Bourdelle, Y. Camenen, and J. Garcia, "Real-time capable first principle based modelling of tokamak turbulent transport," Nuclear Fusion, vol. 55, no. 9, p. 092001, 2015.

[35] F. Felici, J. Citrin, A. Teplukhina, J. Redondo, C. Bourdelle, F. Imbeaux, O. Sauter, J. Contributors, E. M. Team, et al., "Real-time-capable prediction of temperature and density profiles in a tokamak using raptor and a first-principle-based transport model," Nuclear Fusion, vol. 58, no. 9, p. 096006, 2018.

[36] M. Boyer, S. Kaye, and K. Erickson, "Real-time capable modeling of neutral beam injection on nstx-u using neural networks," Nuclear Fusion, vol. 59, no. 5, p. 056008, 2019.

[37] J. Citrin, C. Bourdelle, F. J. Casson, C. Angioni, N. Bonanomi, Y. Camenen, X. Garbet, L. Garzotti, T. Görler, O. Gürcan, et al., "Tractable flux-driven temperature, density, and rotation profile evolution with the quasilinear gyrokinetic transport model qualikiz," Plasma Physics and Controlled Fusion, vol. 59, no. 12, p. 124005, 2017.

[38] D. Moreau, M. L. Walker, J. R. Ferron, F. Liu, E. Schuster, J. E. Barton, M. D. Boyer, K. H. Burrell, S. Flanagan, P. Gohil, et al., "Integrated magnetic and kinetic control of advanced tokamak plasmas on diii-d based on data-driven models," Nuclear Fusion, vol. 53, no. 6, p. 063020,2013

[39] E. A. Wan and R. Van Der Merwe, "The unscented kalman filter for nonlinear estimation," Proceedings of the IEEE 2000 Adaptive Systems for Signal Processing, Communications, and Control Symposium (Cat. No. O0EX373), pp. 153-158, 2000.

[40] F. Felici, M. de Baar, and M. Steinbuch, "A dynamic state observer for real-time reconstruction of the tokamak plasma profile state and disturbances," 2014 American Control Conference, pp. 4816-4823, 2014

[41] T. Blanken, F. Felici, C. Rapson, M. de Baar, W. Heemels, et al., "Control-oriented modeling of the plasma particle density in tokamaks and application to real-time density profile reconstruction," Fusion Engineering and Design, vol. 126, pp. 87-103, 2018.

[42] O. Sauter and S. Y. Medvedev, "Tokamak coordinate conventions: Cocos," Computer Physics Communications, vol. 184, no. 2, pp. 293-302, 2013.

[43] J.-M. Moret, F. Buhlmann, and G. Tonetti, "Fast single loop diamagnetic measurements on the tcv tokamak," Review of Scientific instruments, vol. 74, no. 11, pp. 4634-4643, 2003.

[44] S.Barry, "The extension of the fir interferometer of tcv to a polarimeter and measurements of the faraday rotation caused by the poloidal magnetic field," Univ. of Ireland, PhD thesis, EPFL-Lausanne report LRP 638/99, 1999.

[45] A. Teplukhina, "Realistic multi-machine tokamak profile simulations and numerical ramp-down optimization using the raptor code," PhD thesis No 8478, 2018, EPFL, Switzerland, DOI: 10.5075/epfl-thesis-8478.

[46] F. Felici, T. Blanken, E. Maljaars, H. Van den Brand, J. Citrin, D. Hogeweij, M. Scheffer, 
M. de Baar, M. Steinbuch, S. Coda, et al., "Real-time model-based plasma state estimation, monitoring and integrated control in tcv, ASDEX-upgrade and ITER," Preprint: 2016 IAEA Fusion Energy Conf.(Kyoto, Japan, 1722 October 2016) [EX/P8-33].

[47] O. Sauter, C. Angioni, and Y. Lin-Liu, "Neoclassical conductivity and bootstrap current formulas for general axisymmetric equilibria and arbitrary collisionality regime," Physics of Plasmas, vol. 6, no. 7, pp. 2834-2839, 1999.

[48] O. Sauter, C. Angioni, and Y. Lin-Liu, "Erratum:neoclassical conductivity and bootstrap current formulas for general axisymmetric equilibria and arbitrary collisionality regime," Physics of Plasmas, vol. 9, p. 5140, 2002.

[49] R. E. Kalman, "A new approach to linear filtering and prediction problems," Journal of basic Engineering, vol. 82, no. 1, pp. 35-45, 1960.

[50] D. Simon, "Optimal state estimation: Kalman, h infinity, and nonlinear approaches," John Wiley ES Sons, 2006.

[51] E. Maljaars, F. Felici, T. C. Blanken, C. Galperti, O. Sauter, M. R. de Baar, F. Carpanese, T. P. Goodman, D. Kim, S.-H. Kim, et al., "Profile control simulations and experiments on tcv: a controller test environment and results using a model-based predictive controller," Nuclear Fusion, vol. 57, no. 12, p. 126063, 2017.

[52] H. Arnichand, Y. Andrebe, P. Blanchard, S. Antonioni, S. Couturier, J. Decker, B. Duval, F. Felici, C. Galperti, P.-F. Isoz, et al., "New capabilities of the incoherent thomson scattering diagnostics in the tcv tokamak: divertor and real-time measurements," Journal of Instrumentation, vol. 14, no. 09, p. C09013, 2019.

[53] R. Coelho, D. Alves, N. Hawkes, M. Brix, and J. E. Contributors, "Real-time data processing and magnetic field pitch angle estimation of the jet motional stark effect diagnostic based on kalman filtering," Review of Scientific Instruments, vol. 80, no. 6, p. 063504, 2009.

[54] C. Holcomb, M. Makowski, R. Jayakumar, S. Allen, R. Ellis, R. Geer, D. Behne, K. Morris, L. Seppala, and J. Moller, "Motional stark effect diagnostic expansion on diii-d for enhanced current and e r profile measurements," Review of scientific instruments, vol. 77, no. 10, p. 10E506, 2006.

[55] E. Poli, A. Bock, M. Lochbrunner, O. Maj, M. Reich, A. Snicker, A. Stegmeir, F. Volpe, N. Bertelli, R. Bilato, et al., "Torbeam 2.0, a paraxial beam tracing code for electroncyclotron beams in fusion plasmas for extended physics applications," Computer Physics Communications, vol. 225, pp. 36-46, 2018.

[56] M. Weiland, R. Bilato, R. Dux, B. Geiger, A. Lebschy, F. Felici, R. Fischer, D. Rittich, M. van Zeeland, E. M. Team, et al., "Rabbit: Real-time simulation of the nbi fast-ion distribution," Nuclear Fusion, vol. 58, no. 8, p. 082032, 2018.

[57] R. Fischer, A. Bock, A. Burckhart, O. P. Ford, L. Giannone, V. Igochine, M. Weiland, and M. Willensdorfer, "Sawtooth induced q-profile evolution at ASDEX upgrade," Nuclear Fusion, vol. 59, no. 5, p. 056010, 2019.

[58] B. Kadomtsev, "Magnetic field line reconnection," Reports on Progress in Physics, vol. 50, no. 2, p. $115,1987$.

[59] C. Piron, G. Manduchi, P. Bettini, F. Felici, C. Finotti, P. Franz, O. Kudlacek, G. Marchiori, L. Marrelli, J.-M. Moret, et al., "Integration of the state observer raptor in the real-time marte framework at rfx-mod," Fusion Engineering and Design, vol. 123, pp. 616-619, 2017.

[60] F. Felici, "Real-time control of tokamak plasmas: from control of physics to physics-based control," PhD thesis No 5203, 2011, EPFL, Switzerland, DOI:10.5075/epfl-thesis-5203, 2011.

[61] K. Ogata and Y. Yang, Modern control engineering, vol. 5. Prentice hall Upper Saddle River, NJ, 2010. 